\title{
Comparison of US and Japanese Regulations for Invitro Dissolution and Invivo Bioequivalence Studies
}

\author{
Latif D. Jamadar*, Krishnamurthy Bhat, Yogesh Shirode, \\ Prashant B. Musmade, Syed Sajjad Hussen and N. Udupa
}

Manipal College of Pharmaceutical Sciences, Manipal, Karnataka-576104, India

\begin{abstract}
A regulation with respect to bioequivalence and invitro dissolution of solid oral dosage forms in USA and Japan is summarized and compared. Significant differences in various parameters like dissolution, biowaiver, inclusion- exclusion criteria of subjects in the clinical trials, statistical results were found between two systems. The regulatory experienced gained up to now is studied and compared.
\end{abstract}

Keywords: US FDA; NIHS; Invitro; Biowaiver; Post approval changes; Study reports

Abbreviations: FDA: Food and Drug Administration; NIHS: National Institute of Health Science; CDER: Center for Drug Bioequivalence; API: Active Pharmaceutical Ingredients; BCS: Biopharmaceutical Classification System; NTI: Narrow Therapeutic Index; SUPAC: Scale Up and Post Approval Changes; IR: Immediate Release dosage form; LOD: Limits of Detection; LOQ: Limits of Quantitation

\section{Introduction}

Dissolution study is critical parameter of pharmaceutical dosage forms. It is well recognized that invitro dissolution testing, whether in development relied upon to screen formulation during development and batch to batch quality control (FDA/CDER, 1997). Current International regulatory authorities require that final quality judgment of oral dosage form be based on its invitro dissolution and invivo bioequivalence testing (Rani and Pargal, 2004). Bioequivalence is a term in pharmacokinetics used to assess the expected in vivo biological equivalence of two proprietary preparations of a drug. Bioequivalence (BE) studies are a major component of ANDAs (FDA/CDER, 2003). They verify that the active ingredient in a generic drug product will be absorbed into the body to the same extent and at the same rate as its corresponding reference listed drug (RLD) product. The significance of BE studies is that when two pharmaceutically equivalent products are shown to be bioequivalent, they are judged to be therapeutically equivalent. Therapeutically equivalent products are expected to have the same safety and efficacy profiles, when administered under the conditions listed in the product labeling. This is the basis for the approval and use of generic drug products. The design, performance and evaluation of bioequivalence studies have received major attention from academia, the pharmaceutical industry and health authorities over the past two decades. In this article we would like to provide an overview regarding the invitro dissolution and invivo BE testing regulations of the USFDA and NIHS JAPAN. There are some difference between the regulations of invitro dissolution and invivo BE studies though both the countries have very stringent regulations. Evaluation and Research; BA/BE: Bioavailability and

\section{Regulation for dissolution testing}

Dissolution chapter in the United States pharmacopoeia (USP 30 Dissolution $<711>$ ) is harmonized with the corresponding text of the Japanese Pharmacopoeia (JP $15^{\text {th }}$ Edition). Pharmacopoeias of both the countries have studied and compared, some differences are highlighted below also given in Table 1 and Table 2.

a. 4 types of the apparatus are recommended by the US FDA for dissolution testing. Basket, paddle, reciprocating cylinder and flow through cell are considered as the Apparatus 1, apparatus 2, apparatus 3 and apparatus 4 respectively. Japanese Pharmacopoeia $15^{\text {th }}$ edition does not describe reciprocating cylinder (Apparatus 3 given in USP) for the dissolution test.

b. The dimensions of 1 liter vessel in apparatus 1 (Basket apparatus) of USP and JP are same, but USP also includes additional dimensions for the vessels having capacities 2 liter and 4 liter.

c. For apparatus 1 (Basket) and apparatus 2 (paddle), both pharmacopeias have given same procedure for dissolution of the immediate release dosage forms and extended release dosage form, however USP procedure for the dissolution of the delayed release dosage form in apparatus 1 and apparatus 2 has not accepted by the JP. For delayed release dosage forms, Japanese pharmacopoeia has given $2 \mathrm{hrs}$ test time for the tablets and capsule in acid stage and $1 \mathrm{hr}$ for granules, where as USP has not given the specifications for granules.

d. For demonstrating suitability of individual apparatus the USP has given the apparatus suitability test for all except for apparatus 4. Such type of test has not given by the Japanese pharmacopoeia.

e. There are two types of the interpretations namely Interpretation 1 and Interpretation 2 under the heading 'Interpretation', in Japanese pharmacopoeia. Acceptance criteria given in the Interpretation 1 of Japanese Pharmacopoeia is same as that of given in the Acceptance Table 1, Acceptance Table 2,

${ }^{*}$ Corresponding author: Latif D. Jamadar, Mitraprem Hsg, Soc, Flat No: 1, Plot No: RH 66/1, MIDC, G BLOCK, Chinchwad, Pune-411019 Maharashtra, India, Tel: +919552495853,+919164563678; E-mail: latifsigma@gmail.com

Received February 16, 2010; Accepted March 14, 2010; Published March 14,2010

Citation: Jamadar LD, Bhat K, Shirode Y, Musmade PB, Hussen SS, et al. (2010) Comparison of US and Japanese Regulations for Invitro Dissolution and Invivo Bioequivalence Studies. J Bioanal Biomed 2: 017-022. doi:10.4172/ 1948-593X.1000016

Copyright: (๑) 2010 Jamadar LD, et al. This is an open-access article distributed under the terms of the Creative Commons Attribution License, which permits unrestricted use, distribution, and reproduction in any medium, provided the original author and source are credited.

J Bioanal Biomed
Volume 2(1) : 017-022 (2010) - 017 
Citation: Jamadar LD, Bhat K, Shirode Y, Musmade PB, Hussen SS, et al. (2010) Comparison of US and Japanese Regulations for Invitro Dissolution and Invivo Bioequivalence Studies. J Bioanal Biomed 2: 017-022. doi:10.4172/1948-593X.1000016

Acceptance Table 3 and Acceptance Table 4 of USP. However, in the 'Interpretations 2' of Japanese Pharmacopoeia, NIHS Japan has given acceptance criteria for immediate release dosage forms, extended release dosage forms and delayed release dosage forms. Provided that 'interpretation 1' should be followed when $Q$ value is specified in the individual monograph otherwise Japanese pharmacopoeia recommended 'Interpretation 2'. This type of acceptance criteria has not given in USP, but USP has given a procedure for pooled sample for immediate release dosage form. This procedure is applicable only for immediate release dosage form and for apparatus 1(Basket) and apparatus 2 (Paddle).

f. Separate guidance on dissolution study requirements and testing condition for the drug products containing acidic, neutral, basic, coated products and low solubility drugs is one of the unique character of Japanese BA/ BE guidance. Also there is difference in specification of testing solution, testing time as well as buffers.

\section{Regulation of biowaiver}

\section{FDA SUPAC IR}

The guidance FDA SUPAC IR (FDA/CDER 1997) was first document to establish a consistent and clear policy to establish scale up and post approval changes to immediate release product.FDA SUPAC IR 1995 allows qualification of several types of changes in following parameters:

1) Component and composition

2) Site changes

3) Batch changes

4) Manufacturing

5) Invitro dissolution

6) In vivo bioequivalence studies.

Guideline identifies three 'Level of changes' level 1(unlikely to have any detectable impact on formulation quality and performance), level 2(could have significant impact), level 3 (likely to have a significant impact) (Gupta et al., 2006).

With reference to Table 3 it can be concluded that.

a) Most of level 3 changes require in vivo bioequivalence to be demonstrated.

b) Level 1 change requires only documentation that after the changes drug product still complies with the in vitro dissolution release specification.

c) In level 2 can be qualified by in vitro dissolution release specification which is depends on the three cases shown in Table 3.

One caveat (Gupta et al., 2006) to this guideline Is, This guideline does not give the post approval changes of API containing narrow therapeutic indices drugs (NTI). Narrow therapeutic drug always requires the in vivo bioequivalence qualification testing on each post approval changes. However $21 \mathrm{CFR}$ 320.33 (c) defines it as there is less than 2 fold difference in median lethal dose $\mathrm{LD}_{50}$ and median effective dose $\mathrm{ED}_{50}$ values, or have less than 2 fold difference in the minimum toxic concentration and minimum effective concentration of the drug in the blood.

\section{FDA BCS 2000}

The guidance FDA, BCS (2000) (FDA/CDER 2000) categorizes APIs in to four classes:

Class1: High solubility- high permeability.

Class2: Low solubility- high permeability.

Class3: High solubility- low permeability.

Class4: Low solubility- low permeability.

FDA guideline allows biowaiver for the drug products with BCS class I API as it contain highly soluble and highly permeable drugs like antipyrine, caffeine, carbamazepine but guidance does not accept biowaiver for Class II, III and IV. In addition FDA has given set of requirements for drug product from such class.

1. Drug product must show rapid dissolution, i.e. not less than $85 \%$ labeled amount of the drug substance dissolved within $30 \mathrm{~min}$ in a volume of $900 \mathrm{ml}$ or less in each of the following media: (1) $0.1 \mathrm{~N} \mathrm{HCl}$ or Simulated Gastric Fluid USP without enzymes; (2) a pH 4.5 buffer; and (3) a pH 6.8 buffer or Simulated Intestinal Fluid USP without enzymes.

\begin{tabular}{|l|l|l|}
\hline \multicolumn{2}{|l|}{ LEVEL } & DISSOLUTION TESTINGS \\
\hline Level 1 & None beyond the application or compendial requirements \\
\hline \multirow{3}{*}{ Level 2} & Case 1 (HP \& HS) & $0.1 \mathrm{~N} \mathrm{HCl,}$ \\
\cline { 2 - 3 } & Case 2 (LP \& HS) & $0.1 \mathrm{~N} \mathrm{HCl,} \mathrm{USP} \mathrm{(pH4.5,} \mathrm{6.5,} \mathrm{7.5)}$ \\
\cline { 2 - 3 } & Case 3 (HP \& LS) & Application/compendial medium \\
\hline \multirow{2}{*}{ Level 3 } & Case 1 & Biowaiver of in vivo BE test based on BCS \\
\cline { 2 - 3 } & Case 2, case 3 & Dissolution test and in vivo BE test \\
\hline
\end{tabular}

HP: High Permeability; HS: High solubility, LP: low permeability, LS: low Solubility

Table 1: Dissolution testing in the various Levels of Changes for FDA SUPAC IR 1995.

\begin{tabular}{|l|l|}
\hline LEVEL OF CHANGES & DISSOLUTION TESTINGS \\
\hline Level A & No documentation required. \\
\hline Level B & Dissolution test at pH: $1.2,4.0,6.8$, water. \\
\hline \multirow{2}{*}{ Level C } & Dissolution test at pH: $1.2,4.0,6.8$, water. \\
& If the therapeutic range is narrow or drug is poorly \\
& Soluble, in vivo BE test is required. \\
\hline \multirow{2}{*}{ Level D } & $\begin{array}{l}\text { Dissolution test at } \mathrm{pH}: 1.2,4.0,6.8, \text { water. } \\
\text { If the drug is poorly soluble, in vivo BE test is required. }\end{array}$ \\
\hline \multirow{2}{*}{ Level E } & Dissolution test at pH: $1.2,4.0,6.8$, water. \\
& In vivo BE test is required for all products. \\
\hline
\end{tabular}

Table 2: Dissolution testing in the various Levels of Changes for Japanese Guidance. 


\section{Journal of Bioanalysis \& Biomedicine - Open Access JBABM/Vol.2 Issue 1}

2. Excipients should not affect the rate or extent of absorption of a highly soluble and highly permeable drug substance that is formulated in a rapidly dissolving IR product. This criterion is considered to be met when amount of excipients in drug product is same in that of FDA approved IR dosage form as give in the orange book.

3. For biowaiver, if drug is prodrug and its conversion to active drug is occurring after intestinal permeation then FDA recommends to measure its permeability.

In following cases Biowaiver are not applicable.

1. For Narrow therapeutic range drug

2. Products to be absorbed in oral cavity.

If the drug product complies all of the above conditions then calculation of dissolution of similarity (should be $\geq 50$ ) is sufficient to qualify biowaiver.

\section{FDA BA/BE 2003}

FDA BA/BE 2003 (FDA/CDER, 2003) guidance is another option which give waiver of in vivo bioequivalence test under CFR 320.22 (d) (2) when different strengths are in proportionally similar in active and inactive ingredients to strength of the product. There are three conditions under which drug product can be considered as proportionally similar.

1. All active and inactive ingredients are in exactly same proportion between different strength.

2. If the sum of all differences in excipients between two formulation is within $10 \%$ i.e. falls within level 2 of FDA SUPAC IR 1995

3. For high potency API ( $5 \mathrm{mg}$ dose), when total weight of the strengths is same, same excipients are used and small difference in composition is compensated by one of the excipients.

\section{Regulation in Japan}

No biowaiver accepted under Japanese regulation. For the approval of any generic solid oral immediate release dosage form, in vivo bioequivalence study is compulsory.
Two guidelines guideline produced by the NIHS which relates to Japanese BA-BE these are namely.

1. Guideline for Bioequivalence Studies for Formulation Changes of Oral Solid Dosage Forms (NIHS Japan, 2000).

2. Guideline for Bioequivalence Studies for Different Strengths of Oral Solid Dosage Forms (NIHS Japan, 2000)

Guidelines for Bioequivalence Studies for Formulation Changes of Oral Solid Dosage Forms describe total 5 levels of changes from level A to Level E. Excipients changes which are in trace amounts and does not affect the dissolution profile of the drug comes under Level A, Level A and Level B can be qualified by in vitro dissolution test as per JP, provided that $85 \%$ drug should be dissolved in 30 mins in multiple media. While in vivo BE test along with invitro dissolution test is recommended for the Level C-E. In Level C NTI drugs are exceptions for the in vivo Bioequivalence test if $85 \%$ of the NTI drug from the test NTI product dissolved in multi dissolution media within 30 minutes. The regulation qualification of post approval changes in IR solid oral dosage forms are summarized in Table 4.

Though Japanese guidance addressing formulation changes seems thus to be aligned in several effects with guidance FDA SUPAC IR 1995, there are some differences as described below.

1. Level B-C in the Japanese guideline is corresponds with the FDA SUPAC IR 1995, levels 2-3. However Level D is specific to Japan allowing for a larger change for drug product. Though Level D and Level 3 have same specifications, Level D allows biowaiver only for the Non narrow therapeutic drugs, high solubility drugs and rapidly dissolving drugs.

2. In Japanese guidance, there is separate table on the coat versus coated materials. Total change for the Level B, Level C and Level D is $5 \%, 10 \%$ and $15 \%$ respectively, while in the FDA SUPAC IR 1995 guidance the change for the film coat is of $1 \%$ for the Level 1 and $2 \%$ for the Level 2.

3. Japanese guideline does not accept BCS. It is believed that the formulation changes may be altering the bioavailability

\begin{tabular}{|c|c|c|c|}
\hline \multicolumn{2}{|r|}{ Changes } & \multirow{2}{*}{$\begin{array}{c}\text { Level } \\
\text { Level } 1\end{array}$} & \multirow{2}{*}{$\begin{array}{c}\text { Dissolution Requirements } \\
\text { Dissolution Release Requirements }\end{array}$} \\
\hline \multirow{3}{*}{ Composition } & Total Excipients changes $\leq 5 \%$ & & \\
\hline & Total Excipients changes $\leq 10 \%$ & \multirow[t]{2}{*}{ Level 2} & $\begin{array}{c}f_{2} \text { Dissolution profile similarity in multiple media, unless } \\
\text { Active pharmaceutical ingredients } \mathrm{BCS} 1+\text { drug product } \\
\text { dissolve }>85 \% \text { in } 0.1 \mathrm{~N} \mathrm{HCl} \text { in } 15 \text { min. }\end{array}$ \\
\hline & Total Excipients changes $>10 \%$ & & In vivo Bioequivalence \\
\hline \multirow{3}{*}{ Site } & Within same facility & \multirow{3}{*}{$\begin{array}{l}\text { Level } 1 \\
\text { Level } 2 \\
\text { Level } 3\end{array}$} & Dissolution Release Requirements \\
\hline & Within same campus & & Dissolution Release Requirements \\
\hline & Different campus & & $f_{2}$ dissolution profile similarity in filed medium \\
\hline \multirow{2}{*}{ Batch Size } & Up to $10 \mathrm{x}$ & \multirow{2}{*}{$\begin{array}{l}\text { Level } 1 \\
\text { Level } 2\end{array}$} & Dissolution Release Requirements \\
\hline & Beyond 10x & & $f_{2}$ dissolution profile similarity in filed medium \\
\hline \multirow[b]{2}{*}{ Equipment } & Automation of transfer to alternate equipment & \multirow[b]{2}{*}{$\begin{array}{l}\text { Level } 1 \\
\text { Level } 2\end{array}$} & Dissolution Release Requirements \\
\hline & Different design or operating principle & & $f_{2}$ dissolution profile similarity in filed medium \\
\hline \multirow[b]{3}{*}{ Process } & Changes within field and validated ranges & \multirow{2}{*}{ Level 1} & Dissolution Release Requirements \\
\hline & Changes within field and validated ranges & & $f_{2}$ dissolution profile similarity in filed medium \\
\hline & Major changes & $\begin{array}{l}\text { Level } 2 \\
\text { Level } 3\end{array}$ & In vivo Bioequivalence \\
\hline
\end{tabular}

Table 3: US regulation for qualification of post approval changes in IR solid oral dosage forms.

J Bioanal Biomed $\quad$ ISSN:1948-593X JBABM, an open access journal

Volume 2(1) : 017-022 (2010) - 019 
Citation: Jamadar LD, Bhat K, Shirode Y, Musmade PB, Hussen SS, et al. (2010) Comparison of US and Japanese Regulations for Invitro Dissolution and Invivo Bioequivalence Studies. J Bioanal Biomed 2: 017-022. doi:10.4172/1948-593X.1000016

\begin{tabular}{|c|c|c|}
\hline Changes & Level & Qualification \\
\hline Ratio of component Material & A & Compliance to in vitro Dissolution Specification \\
\hline $\begin{array}{l}\text { Addition or Deletion of Trace } \\
\text { Excipients }\end{array}$ & A & Compliance to in vitro Dissolution Specification \\
\hline Total Change in excipients $\leq 5 \%$ & $\mathrm{~B}$ & $F_{2}$ dissolution profile similarity in multiple Media \\
\hline Total Change in excipients $\leq 10 \%$ & $\mathrm{C}$ & $\begin{array}{l}\text { a. } F_{2} \text { dissolution profile similarity in multiple Media for non NTI API and for NTI API showing } \geq 85 \% \\
\text { dissolution within } 30 \text { min. } \\
\text { b. In vivo BE study for API having low solubility. } \\
\text { c. In vivo BE study for NTI API having low solubility and showing }<85 \% \text { dissolution within } 30 \text { min }\end{array}$ \\
\hline Total Change in excipients $\leq 15 \%$ & $\mathrm{D}$ & $\begin{array}{l}\text { a. } F_{2} \text { dissolution profile similarity in multiple Media for Non NTI API showing } \geq 85 \% \text { dissolution within } \\
30 \mathrm{~min} \text {. } \\
\text { b. In vivo BE study for NTI API. }\end{array}$ \\
\hline
\end{tabular}

Table 4: Japanese regulation for qualification of post approval changes in IR solid oral dosage forms.

of the dosage form rather than the solubility and permeability of the drug products. Also bioequivalence of most of the IR products is mainly assured by the dissolution testing in multiple media hence BCS classification (based on the solubility and permeability) is not important.

\section{Number of subject and subject selection criteria}

FDA BA/BE 2003 guidance and food effect BA/ fed BE guidance recommended minimum 12 number of subject (more than $18 \mathrm{yrs}$ old) for invivo BE study. While Japanese guidance recommended total 20 number of the subjects to be included in the BE study. Also for add on study additional 10 subjects are recommended along with initial subjects (NIHS Japan, 2000).

The inclusion and exclusion criteria are almost same for both the countries, While Japanese guidance given additional recommendation of inclusion of the subjects having low gastric acidity (Achlorhydric subjects) for the reference and test product having significant difference at around $\mathrm{pH} 6.8$ or between $\mathrm{pH} 3$ and $\mathrm{pH} 6.8$ for the product containing basic drugs. However this rule is not applied for the enteric coated product.

\section{Study}

\section{Regulation in US}

FDA BA BE 2003 has given option of pilot study. Sponsor can carry out pilot study on small number of the subjects before proceeding to BE study and this study can be used to validate analytical methodology, optimize sample collection time interval and provide other information.

In the bioequivalence study, for food effect study FDA BA BE recommended that

a. Test and reference product should be administered with 240 ml water

b. No food should be allowed at least for 4 hrs post dose.

c. Abstain from alcohol for $24 \mathrm{hrs}$ before each study period and until after the last sample from each period is collected.

d. In sample collection and sampling time 12-18 samples including predose sample should be collected per subject per dose.

e. If the predose concentration is $>5$ percent of $C_{\text {max }}$ value then FDA BA BE 2003 recommended to drop out that subject from the study.

f. FDA BA BE 2003 recommend that data from subjects who experience emesis during the course of a $\mathrm{BE}$ study for immediate-release products be deleted from statistical analysis if vomiting occurs at or before 2 times median $\mathrm{T}_{\max }$.

\section{Food-Effect Bioavailability and Fed Bioequivalence Studies 2002}

Guideline (FDA/CDER, 2002) recommended that Fed BE study is unnecessary for post approval changes in the dosage form where only in vivo re-documentation of $\mathrm{BE}$ recommended under fasting condition.

\section{Recommendation for Test Meal (NIHS Japan, 2000)}

General principle behind the design of test meal is to provide greatest effect on GI physiology so that systemic drug availability is affected maximally. A high fat and a high caloric meal is recommended as test meal for food effect BA study and Fed BE study. Fat should be $50 \%$ of total caloric content of the meal and 800 to 1000 calories considered as high calories. As per US regulation meal should contain 150 calories of protein, 250 calories of carbohydrates and 500-600 calories of fat.

It is recommended that the sponsor should report the caloric breakdown of the test meal during the study and also given that sponsor should provide rationale for the breakdown. FDA food effect BA study and Fed BE guideline 2002 gives option to sponsor to choose meal of different combination of fat, carbohydrate and protein as given in guidance 'food effect BA and fed BE 2002' but guidance also recommends that sponsor should choose one of meal containing high fat and high calories.

For fasting study guideline recommended overnight fasting of $10 \mathrm{hrs}$ and no food should be allowed for $4 \mathrm{hrs}$ after dose. For fed treatment guideline recommended fasting of $10 \mathrm{hrs}$ subject should start recommended meal in 30 min or less; however the drug product should be administered 30 after the start of meal.

Though Japanese regulation addressing type of the studies is aligned in same manner as that of the US regulations, there are some difference in the sample collection, test meal, dose administration etc. These are listed below:

1. FDA food effect BA and fed BE study guideline recommended that both fasting study and fed study is required for the drug product while Japanese guidance specifies condition under which fed study need to be carried out.

2. FDA BCS 2003 gave a very good option to the sponsor that is 'Pilot study'. This may be useful for the sponsor to validate analytical method, to assess the variability also to optimize sample collection intervals. For example in the modified re-

J Bioanal Biomed 


\section{Journal of Bioanalysis \& Biomedicine - Open Access \\ JBABM/Vol.2 Issue 1}

\begin{tabular}{|l|c|c|}
\hline Parameters & US Regulation & Japanese Regulation \\
\hline Total Energy from Meal & $800-1000$ calories & $700 \mathrm{kcal}$ \\
\hline \multirow{2}{*}{ Energy from Fat } & a. $\quad$ High fat and high caloric Meal & $\begin{array}{l}\text { a. } \\
\text { bow fat and high caloric meal. } \\
20 \% \text { energy of total Meal }(140 \mathrm{kcal})\end{array}$ \\
\hline Time Limits & b. $50 \%$ of total meal $(500-600 \mathrm{cal})$ & $20 \mathrm{~min}$ \\
\hline
\end{tabular}

Table 5: Difference in the Test meal and Energy of test meal between both the countries.

lease dosage form careful pilot study may be useful to determine the sampling schedule to assess the lag time and dose dumping.

3. FDA BCS 2003 recommended that drug should be administered with 8 ounce means $240 \mathrm{ml}$ of the water while NIHS (Japanese) BA-BE guideline recommended that drug should be administered with the $100-200 \mathrm{ml}$ of water, generally $150 \mathrm{ml}$.

\section{Test meal and Energy of test meal}

1. In NIHS (Japanese) guidance the low fat and high caloric food is recommended. The caloric content is approximately $700 \mathrm{kcal}$ out of which not more than $20 \%$ (140 kcal) is derived from the fat (Table 5) (NIHS Japan, 2000).

2. FDA food effect BA and Fed BE guidance 2002 given the contents limits of the fat, carbohydrates and proteins. NIHS (Japanese) guidance has not given the contents of the carbohydrates as well as proteins.

3. FDA food effect BA and Fed BE guidance 2002 given 30 min time limit to eat the meal while NIHS Japan guidance given 20 min time limit to eat meal. While both guidance recommended 30 minutes to administered the drug after start of the meal.

4. FDA food effect BA and Fed BE guidance 2002 gives an example of the test meal which help to sponsor to choose the test meal of appropriate energy while NIHS Japanese has not given any example of the meal.

\section{Difference in statistical calculations and bioequivalence re- port submission between US FDA and NIHS Japan}

Though the regulation addressing the statistical outcomes and parameters submitted in bioequivalence reports, between both the countries is aligned on same principles but there are some differences which are as follows:

\section{Statistical calculations:}

a. Japanese guidance given the $90 \%$ shortest confidence interval or two- one sided t test with significant level of 5\% should be used.FDA BA BE 2003 also recommended the $90 \%$ shortest confidence interval for the Average bioequivalence .For the population BE and Individual BE, FDA guidance recommends $95 \%$ upper confidence bound for population BE or equivalently $95 \%$ upper bound confidence for a linearized form of population $\mathrm{BE}$.

b. FDA BA BE guidance and FDA statistic approach guidance (FDA/ CDER 2001) given traditional BE limit 80-125\% for non-narrow therapeutic range and this criteria same for the narrow range therapeutic drugs. Japanese guidance recommends same criteria for the solid dosage forms but also given 3 exceptional cases under which drug product can be considered as bioequivalent with the reference product though it does not comply with above range. These exception are-

i. Sample size of more than 20 including pooled sample size (initial sample and add on sample) is not less than 30 .

ii. Difference in the logarithmic values of AUC and $\mathrm{C}_{\text {max }}$ of test and reference products are between $[\log 0.9, \log 1.11]$

iii. Equivalent dissolution conditions of test and reference products.

\section{Details to be submitted in bioequivalence report:}

USFDA recommended following points in the bioequivalence report submission, Japanese guidance have not given these points in the guideline.

a. Bioanalytical method validation information-LOD, recovery, LOQ, Precision study, stability study, dilution integrity.

b. Formulation data: Cores and coating information

c. Reanalysis of study samples with limit of quantification and reasons of reanalysis.

d. Subject dropout information with reason provided with replaced subject information.

e. Standard curve and QC data

f. SOP's dealing with bioanalytical repeats of study samples.

\section{Conclusion}

It is our hope that, this review will provide an easy quick overview for comparing several parameters of the dissolution studies and bioequivalence regulations of the United States and Japan. It will also useful for the quick reference of the regional or scientific philosophy on their applicability We caution that the regulatory landscape is dynamic, and this review is no substitute for consultation with the full text of currently applicable guidances and regulations, or indeed for direct consultation with the Health Authorities when biowaivers are being considered as part of registrational strategy.

It is further hoped that continued research and scientific/regulatory dialog might lead to optimal and harmonized application of biowaiver principles in the interest of improved worldwide access to needed medicines.

\section{References}

1. FDA (1995) Guidance for industry: Immediate release solid oral dosage forms: Scale Up and post approval changes: Chemistry, Manufacturing and control US Food and drug administration center for evaluation of drug and research. »CrossRef » PubMed » Google Scholar

2. FDA (2000) Guidance for Industry: Waiver of invivo Bioavailability and bioequivalence studies for immediate release dosage forms based on biopharmaceutical classification system. US Food and drug administration center for evaluation of drug and research. »CrossRef » PubMed » Google Scholar

3. FDA (2001) Guidance for Industry: Statistical Approaches to Establishing Bioequivalence US Food and drug administration center for evaluation of drug and research. »CrossRef » PubMed » Google Scholar

4. FDA (2001) Guidance for Industry Statistical Approach to establishing bioequivalence studies. US Food and drug administration center for evaluation 
Citation: Jamadar LD, Bhat K, Shirode Y, Musmade PB, Hussen SS, et al. (2010) Comparison of US and Japanese Regulations for Invitro Dissolution and Invivo Bioequivalence Studies. J Bioanal Biomed 2: 017-022. doi:10.4172/1948-593X.1000016

of drug and research. » CrossRef » PubMed » Google Scholar

5. FDA (2002) Guidance for Industry Food-Effect Bioavailability and Fed Bioequivalence Studies. US Food and drug administration center for evaluation of drug and research. » CrossRef » PubMed » Google Scholar

6. FDA (2003) Guidance for industry: Bioavailability and Bioequivalence Studies for Orally Administered Drug Products General Considerations US Food and drug administration center for evaluation of drug and research, Cited on 21 September 2009. » CrossRef » PubMed » Google Scholar

7. Formatting of Bioequivalence summary tables (2009) US Food and drug administration center for evaluation of drug and research. »CrossRef » PubMed » Google Scholar

8. Guidance for industry, dissolution testing for immediate solid oral dosage forms, US department of health and human services, food and drug administration, center for drug evalution and research, Aug 1997. » CrossRef » PubMed » Google Scholar

9. Gupta E, Barends DM, Yamashita E, Lentz KA, Harmsze AM, et al. (2006)
Review of global regulation concerning biowaiver for immediate release solid dosage forms. Eur J Pharm Sci 29: 315-324. » CrossRef » PubMed » Google Scholar

10.Japanese Pharmacopoeia; Ministry of health and labor welfare. Fifteenth Edition 116-119. »CrossRef » PubMed » Google Scholar

11.NIHS (1997) Guideline for Bioequivalence Studies of Generic Products. National Institute of Health Sciences, Japan. » CrossRef » PubMed » Google Scholar

12.NIHS (2000a) Guideline for Bioequivalence Studies for Different Strengths of Oral Solid Dosage Forms. National Institute of Health Sciences, Japan. » CrossRef » PubMed » Google Scholar

13.NIHS (2000b) Guideline for Bioequivalence Studies for Formulation Changes of Oral Solid Dosage Forms. National Institute of Health Sciences, Japan. »CrossRef » PubMed » Google Scholar

14.Rani S, Pargal A (2004) Bioequivalence: An overview of statistical concepts. Indian J Pharmacol 36: 209-216. »CrossRef » PubMed » Google Scholar

15.United State Pharmacopoeia 30 National formulary 25; Published by Rockwille mayrland USA. < 711> dissolution 2411-2414. » CrossRef » PubMed » Google Scholar 